\title{
Intermittent Oroesophageal Tube Feeding via the Airway in Patients With Dysphagia
}

\author{
Hyo Kyung Shin, $\mathrm{MD}^{1}$, Kyo In Koo, $\mathrm{PhD}^{2}$, Chang Ho Hwang, MD, PhD ${ }^{1}$
}

\begin{abstract}
${ }^{1}$ Department of Physical Medicine and Rehabilitation, Ulsan University Hospital, University of Ulsan College of Medicine, Ulsan; ${ }^{2}$ School of Electrical Engineering, University of Ulsan, Ulsan, Korea
\end{abstract}

\begin{abstract}
Objective To investigate the feasibility of the use of the oropharyngeal airway (OPA) during intermittent oroesophageal tube (IOET) feeding.

Methods Ten patients, who were evaluated using the videofluoroscopic swallowing study (VFSS), were enrolled. One patient withdrew from the study during the study period. Tube insertion time with and without OPA use was recorded in the same patients in a random order during the VFSS. Patients who could safely undergo IOET feeding were then randomly allocated to 2 groups (OPA and non-OPA). Satisfaction Questionnaire with Gastrostomy Feeding (SAGA-8) scores and pneumonia incidence were assessed on the 3rd and 10th day after the VFSS. Nonparametric analysis was used for statistical analyses.

Results The IOET insertion time was significantly shorter in the OPA group than in the non-OPA group (17.72 \pm 5.79 vs. $25.41 \pm 10.41$ seconds; $\mathrm{p}=0.017$ ). Complications were not significantly different between the 2 groups $(\mathrm{p}=0.054)$. Furthermore, although there were no significant differences in the SAGA-8 scores $(25.50 \pm 2.38$ vs. 21.40 \pm 3.13 ; $\mathrm{p}=0.066$ ), which reflect the patient/caregiver satisfaction and the ease of tube insertion, patients in the OPA group tended to be more satisfied with the feeding procedure.

Conclusion Although the small size of the study cohort is a limitation of our study, the use of the OPA appears to be beneficial during IOET feeding in patients with dysphagia.
\end{abstract}

Keywords Dysphagia, Intermittent oroesophageal tube, Swallowing, Videofluoroscopy

\footnotetext{
Received December 11, 2015; Accepted March 16, 2016

Corresponding author: Chang Ho Hwang

Department of Physical Medicine and Rehabilitation, Ulsan University Hospital, University of Ulsan College of Medicine, 877 Bangeojinsunhwan-doro, Dong-gu, Ulsan 44033, Korea. Tel: +82-52-250-7210, Fax: +82-52-250-7211, E-mail: chhwang1220ciba@gmail.com

ORCID: Hyo Kyung Shin (http://orcid.org/0000-0003-0714-0850); Kyo In Koo (http://orcid.org/0000-0003-4173-9218); Chang Ho Hwang (http://orcid. org/0000-0003-0444-3602).
}

() This is an open-access article distributed under the terms of the Creative Commons Attribution Non-Commercial License (http://creativecommons.org/ licenses/by-nc/4.0) which permits unrestricted noncommercial use, distribution, and reproduction in any medium, provided the original work is properly cited. Copyright $\odot 2016$ by Korean Academy of Rehabilitation Medicine 


\section{INTRODUCTION}

Dysphagia may lead to aspiration pneumonia, malnutrition, or dehydration [1]. Hence, an appropriate alternative feeding method is required in patients with dysphagia. The usual methods for nutritional support include parenteral and enteral nutrition. As the parenteral route cannot provide sufficient nutrition and is associated with the risk of bacterial infection, enteral nutrition is considered to be more suitable for patients with an intact intestinal tract. Common enteral feeding methods include nasogastric tube use and percutaneous endoscopic gastrostomy. Since continuous feeding via a nasogastric tube reportedly leads to complications such as inflammation, restriction of oropharyngeal movement, gastro-esophageal reflux, diarrhea, and aspiration pneumonia, short-term use of this method is recommended [2]. Although percutaneous endoscopic gastrostomy is relatively safe and can be used for long-term nutritional support, this procedure is invasive and can be associated with complications such as bleeding, infection, mucosal ulcer, diarrhea, and reflux [3].

Intermittent oroesophageal tube (IOET) feeding was first used in 1985 for nutritional support in children with dysphagia [2]. Its subsequent application in stroke patients reduced the incidence of complications such as pneumonia, diarrhea, and reflux $[2,4,5]$. However, IOET feeding can only be used in alert patients; in unconscious patients, it can lead to complications [6]. In particular, patient participation is vital during tube insertion, necessitating clear consciousness. Consequently, it is difficult to perform IOET feeding in patients who do not properly understand how to insert the tube and in stroke patients with upper limb weakness, which limits the use of this method. Nevertheless, the nasogastric feeding tube can be replaced in cases where a caregiver inserts the IOET. However, blind insertion is associated with the risk of aspiration pneumonia in unconscious patients, as the tube can be placed into the larynx or its insertion can lead to torsion within the pharynx $[6,7]$.

It may be emphasized that the use of an airway device to insert the IOET might ensure rapid and safe insertion and feeding, which could consequently increase the satisfaction of caregivers and patients. However, to the best of our knowledge, no prior study has described the effects of airway device use during IOET feeding. In the present study, we aimed to evaluate the usefulness of a prefabricated oropharyngeal airway (OPA or Guedel airway; Bio-Medical Instruments, Clinton Township, MI, USA) [8] in IOET feeding, even in unconscious patients, and to determine whether the use of the OPA causes a significant change in satisfaction with IOET among dysphagic patients.

\section{MATERIALS AND METHODS}

\section{Subjects}

For this prospective, single-center case series study on the feasibility of oral tube feeding, we enrolled in-patients who were referred to the rehabilitation medicine department of a tertiary hospital for evaluation with a videofluoroscopic swallowing study (VFSS) from June 2014 to February 2015. Test diets, which included 2 and $5 \mathrm{~mL}$ of diluted barium ( $40 \%$ weight/volume), curd-type yogurt, and boiled rice, were administered orally to the patients by a physiatrist. Thin fluid $(40 \% \mathrm{w} / \mathrm{v})$ was used in the OET to reduce the occurrence of aspiration pneumonia and to increase the reliability of the VFSS $[9,10]$. OET feeding was indicated for dysphagic patients with impaired unilateral or bilateral gag reflex, and for those in whom safe oral feeding was not possible. All patients had stable vital signs and could cooperate with their caregivers, who were appropriately trained to perform IOET feeding. Cases in which oral feeding was possible during the VFSS, tube insertion through the esophagus was challenging owing to an osteophyte or cricopharyngeal incoordination, esophageal peristalsis was insufficient or reflux was observed, or informed consent was not obtained were excluded. This study was approved by the ethics committees of clinical research (No. 2014-05-015) and it was performed after obtaining written informed consent from the patients or their legal guardians. Accurately positioning the IOET in the esophagus, rather than in the respiratory tract, is more important than quickly performing the IOET procedure in patients with a decreased level of consciousness and impaired cognition. Although the primary end point was not IOET insertion in a short time but rather safe insertion, no reference about the safety assurance of the procedure could be cited because this is the first study on this topic. In addition, the study would have demonstrated better results if the safety assurance of the OPA had been verified in the pilot study. How- 
ever, it was difficult to verify the safety of the procedure. Thus, the OPA was used instead in a pilot test involving 6 patients, with $5 \%$ significance level and $80 \%$ statistical power, in order to estimate the time required for IOET insertion. It was found that 31 patients were required for the present study. For calculation of the total sample size, the repeated measures ANOVA between factors was used together with the mean value from $\mathrm{G}^{*}$ Power (ver. 3.1, www.psycho.uni-duesseldorf.de), a program used to calculate the number of samples, by applying 0.75 for effect size, 0.05 for a value, 0.9 for power, 3 for number of groups, 4 for number of measurements and 0.769 for correlation of repeated measures, and this showed that a total sample size of 24 patients was needed. In addition, a dropout rate was set at $30 \%$; hence, that the total required number $\mathrm{N}$ was determined to be 31 patients. For continuous variables, the Kolmogorov-Smirnov test was performed to test for normality, and transposition of the log was performed for variables that were not normally distributed (Fig. 1).

\section{Assessments}

Medical history of all subjects was analyzed after classifying the underlying diseases as central nervous system disorders, motor neuron disorders, peripheral nervous system conditions, naso-laryngopharyngeal lesions, and others diseases. Gross cognition was evaluated using the
Mini-Mental State Examination (MMSE) [11]. The level of consciousness was determined as $0,1,2$, or 3 according to the National Institutes of Health Stroke Scale (NIHSS1a). Furthermore, chest radiography was performed to detect underlying aspiration pneumonia. A single researcher performed medical history analysis, MMSE and NIHSS assessment, and chest radiography before VFSS. The MMSE results and NIHSS-1a level of consciousness ratio were evaluated prior to the VFSS, and accordingly, the VFSS was performed; during the examination, the IOET insertion time was assessed by using non- parametric analysis. MMSE is only a screening tool for cognition, and NIHSS is used to assess generalized neurologic deficit after stroke; hence, assessment of consciousness with these two measures might not be adequate. MMSE and NIHSS were used because they were the criteria used to measure the level of consciousness in our hospital although they are crude variables, and there was not enough time for separating them more elaborately.

VFSS was performed at the same location and on the same day. The inter-rater variation in VFSS results was found to be $>90 \%$ [10]. To avoid errors resulting from interference with nasogastric feeding tubes, these tubes were removed 20 minutes before the test. Moreover, in cases of tracheostomy, tests were performed after removal of oral secretions and decompression of the balloon. Physician A, who specialized in dysphagia treatment,

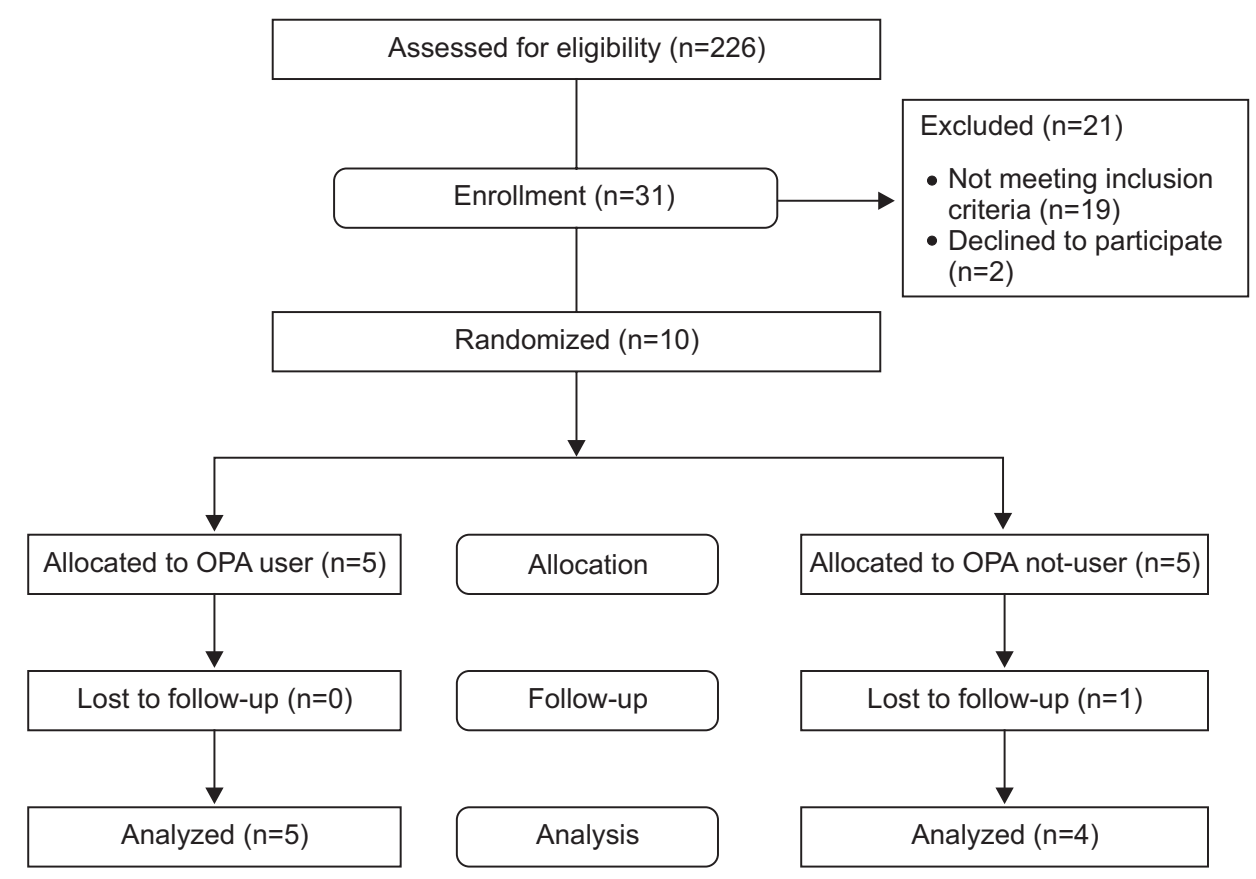

Fig. 1. A flowchart illustrating the number of participants enrolled, allocated to each study group, followed up, and analyzed. OPA, oropharyngeal airway. 
followed the modified Logemann protocol [12] for VFSS with the patient in the lateral position and performed fluoroscopy to determine the exact anatomical structures. Before participating in the VFSS, physician B surveyed the patients for information on age, sex, cause of dysphagia, MMSE, and NIHSS-1a level of consciousness. Before the VFSS, physician C used the random assignment generator (Wichmann-Hill random number generator; http:// www.randomization.com) to assign patients into the OPA and non-OPA groups, at a 1:1 allocation ratio. If it was determined that the patient required an IOE tube, a sealed envelope with the allocation information was delivered to physician A after the VFSS. During the VFSS, if physician A determined that the patient needed an IOE tube, a 3-minute break was allowed during the examination. Then, without viewing the fluoroscopy screen, the use of an OPA was decided via a coin toss, before physician A inserted the IOE tube. Physician B used the X-ray fluoroscopy screen to determine the IOE tube insertion time (i.e., the time taken by the tube tip to advance through the OPA, pass the upper esophageal sphincter, and to be confirmed as being safely inserted into the esophagus), the patient's compliance (i.e., refusing or accepting the procedure), whether the IOE tube protruded into the airway, the torsion of the larynx, and whether the insertion was likely to fail. Then, for patients in whom IOE tube insertion was decided, physician A provided caregiver training and began IOE tube insertion according to the patient's random allocation to either of the two groups (OPA vs. non-OPA) (Fig. 2). OET feeding was attempted in the ward 3 times a day. As a fluoroscope was not available in the ward, physician B guided the patients. Physician B also educated the caregivers and the patients regarding the safe methods for IOET feeding and tube insertion. Af- ter 3 days, physician C conducted a satisfaction survey by administering the Satisfaction Questionnaire with Gastrostomy Feeding (SAGA-8) [13,14] (Appendix 1) directly to the patients if they were fully conscious and were able to communicate clearly or to the caregivers. Physician B determined the reason(s) for cessation of tube feeding (if needed), time at which oral feeding became feasible, and occurrence of complications (diarrhea, aspiration pneumonia). In addition, when the patients were discharged or allowed to start oral feeding, SAGA-8 was administered, reason(s) for cessation of IOET feeding were identified, and complications were recorded once again. Based on this information, we aimed to develop a customized method for easy IOET insertion and to create a set of detailed instructions for the feeding procedure. The protocols for IOET feeding are described in Appendices 2 and 3.

\section{Statistical analyses}

Physician D, who was not aware about the details of the OPA and non-OPA groups, performed the statistical analysis using SPSS ver. 21.0 for Windows (IBM, Armonk, NY, USA). The significance level ( $\alpha$ ) was set at 0.5 , and p-values $<0.05$ were considered to indicate statistical significance. The statistical power was set at $80 \%$, and the expected dropout rate was $30 \%$.

Repeated measures analysis of variance (ANOVA) in combination with the mean value from the software G*Power (ver. 3.1, www.psycho.uni-duesseldorf.de) that calculates the number of samples was used to determine the total sample size. The following values were used: effect size, 0.75; value, 0.05; power, 0.9; number of groups, 3; number of measurements, 4; correlation of repeated measures, 0.769 . This analysis yielded a sample size of
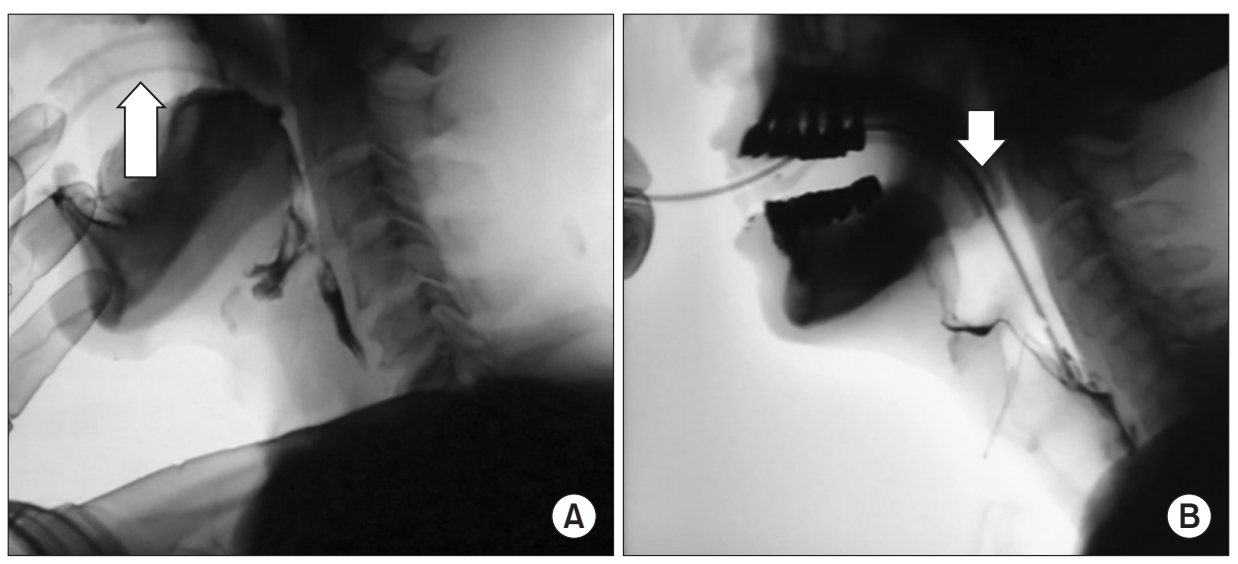

Fig. 2. (A) The oropharyngeal airway (white arrow) was first inserted into the oropharynx, and the patient was asked to bite on it lightly. (B) The intermittent oroesophageal tube (short white arrow) was then inserted as far as possible up to the upper esophageal sphincter. 
24 patients. Considering a dropout rate of $30 \%$, the total required number of patients was determined to be 31 patients.

The Kolmogorov-Smirnov test was performed for continuous variables to test for normality, and translation of the log was performed for variables that were not normally distributed. Based on OPA insertion, a two-sample paired t-test was performed to compare the IOET insertion time. The chi-square test was used for assessing patient compliance, IOET progression into the esophagus, laryngeal torsion, and failure of the IOET procedure, as they were categorical variables. The MMSE results and NIHSS-1a level of consciousness were evaluated prior to performing VFSS. During the examination, the IOET insertion time was assessed by using non-parametric analysis. The MMSE and NIHSS were used because they constituted the tools for measuring consciousness at our hospital. Although they provide relatively crude estimates, there was no time for a more accurate analysis. The SAGA- 8 scores, which were recorded 3 days after the completion of VFSS and at the time of discharge or initiation of oral feeding, were considered ordinal variables and were tested by the Mann-Whitney U test.

Table 1. Demographic characteristics of the subjects

\begin{tabular}{lc}
\hline \multicolumn{1}{c}{ Characteristic } & Value \\
\hline Mean age (yr) & $69.41 \pm 12.05(48-89)$ \\
Sex (male:female) & $8: 1$ \\
Underlying disease & $8(88.9)$ \\
Stroke & $0(0)$ \\
Pneumonia & $1(11.1)$ \\
Other & 1.22 \\
\hline Level of consciousness (NIHSS-1a) & $1(11.1)$ \\
0 & $5(55.6)$ \\
1 & $3(33.3)$ \\
2 & \\
MMSE & $4(44.5)$ \\
$0-10$ & $4(44.5)$ \\
$11-20$ & $1(11.1)$ \\
\hline
\end{tabular}

Values are presented as mean \pm standard deviation (range) or number of subjects (\%).

NIHSS, National Institutes of Health Stroke Scale; MMSE, Mini-Mental State Examination.

\section{RESULTS}

\section{Characteristics of the study subjects}

The mean age of the 9 patients ( 8 men and 1 woman) was $69.41 \pm 12.05$ years. Eight patients experienced stroke, and 1 patient was in poor condition because of pneumonia. One patient had a NIHSS-1a score of 0,5 patients had NIHSS-1a scores of 1 , and 3 patients had NIHSSla scores of 2 . Moreover, 1 patient had mild cognitive impairment (MMSE, 21-26), 4 patients had moderate cognitive impairment (MMSE, 11-20), and 4 patients had severe cognitive impairment (MMSE, 0-10). One patient was suspected to have aspiration pneumonia prior to the VFSS, and 8 patients had no abnormalities on chest radiography (Table 1).

\section{Effects of the use of an OPA for tube insertion}

A significant difference in the time of passage of the IOET from the oral cavity to the upper part of the esophagus was observed depending on whether the OPA was used (non-OPA vs. OPA: $25.41 \pm 10.41$ vs. $17.72 \pm 5.79$ seconds; $\mathrm{p}=0.017)$. Thus, the use of an OPA reduced the IOET insertion time by $7.68 \pm 7.01$ seconds $(p=0.011)$. However, the use of the OPA did not lead to significant differences in the time required for the IOET to reach the oral cavity-pharynx-upper part of the esophagus between groups classified according to the MMSE and NIHSS-1a scores ( $\mathrm{p}>0.05)$. Moreover, no significant relationship was noted between complications such as tube torsion during IOET insertion and the use of the OPA ( $>>0.05$ ) (Tables $2,3)$. In a chest simple X-ray to the VFSS, if there were no other particular considerations, tube insertion was performed more quickly by using an OPA ( $\mathrm{p}=0.019)$. Furthermore, as long as there were no problems with tube insertion into the larynx, the procedure was performed more rapidly and accurately when an OPA was used $(\mathrm{p}=0.033)$.

Table 2. Comparison of the functional transit time

\begin{tabular}{ccc}
\hline & Value & p-value \\
\hline Transit time & & \\
With the OPA & $17.72 \pm 5.79$ & 0.017 \\
Without the OPA & $25.41 \pm 10.41$ & \\
\hline
\end{tabular}

Values are presented as mean \pm standard deviation. OPA, oropharyngeal airway. 
Table 3. Relationships between transit time and level of consciousness, MMSE score, sensations in the oral cavity, and underlying diseases

\begin{tabular}{|c|c|c|}
\hline & \multicolumn{2}{|c|}{ Transit time } \\
\hline & With OPA & Without OPA \\
\hline \multicolumn{3}{|l|}{ NIHSS-1a } \\
\hline 0 & 17.39 & 29.92 \\
\hline 1 & $14.71 \pm 4.25$ & $19.49 \pm 4.81$ \\
\hline 2 & $20.82 \pm 6.80$ & $30.20 \pm 13.49$ \\
\hline $\mathrm{p}$-value & 0.435 & 0.322 \\
\hline \multicolumn{3}{|l|}{ MMSE } \\
\hline $21-26$ & 17.39 & 29.92 \\
\hline $10-20$ & $17.77 \pm 8.09$ & $23.21 \pm 8.69$ \\
\hline$<10$ & $17.75 \pm 4.88$ & $26.48 \pm 14.10$ \\
\hline p-value & 0.875 & 0.717 \\
\hline \multicolumn{3}{|l|}{ Cause of dysphagia } \\
\hline Stroke (brain lesion) & $17.34 \pm 6.06$ & $25.63 \pm 11.11$ \\
\hline Motor neuron disease & NA & NA \\
\hline $\begin{array}{l}\text { Peripheral neuropathy } \\
\text { (DM, etc.) }\end{array}$ & NA & NA \\
\hline Head and neck cancer & NA & NA \\
\hline Malnutrition & 20.77 & 23.65 \\
\hline p-value & 0.439 & 0.699 \\
\hline \multicolumn{3}{|c|}{ Abnormal chest radiography } \\
\hline $\mathrm{O}$ & 23.04 & 46.39 \\
\hline $\mathrm{X}$ & $17.06 \pm 5.81$ & $22.78 \pm 7.29$ \\
\hline $\mathrm{p}$-value & 0.667 & 1.000 \\
\hline \multicolumn{3}{|l|}{ Passage to the larynx } \\
\hline $\mathrm{O}$ & $18.00 \pm 4.76$ & $38.16 \pm 11.65$ \\
\hline $\mathrm{X}$ & $17.58 \pm 6.66$ & $21.76 \pm 7.23$ \\
\hline p-value & 0.905 & 0.0111 \\
\hline \multicolumn{3}{|l|}{ Failure of tube insertion } \\
\hline $\mathrm{O}$ & NA & NA \\
\hline $\mathrm{X}$ & $17.72 \pm 5.79$ & $25.41 \pm 10.41$ \\
\hline p-value & NA & NA \\
\hline \multicolumn{3}{|l|}{ Torsion of the tube } \\
\hline $\mathrm{O}$ & $21.38 \pm 2.35$ & $34.95 \pm 16.18$ \\
\hline $\mathrm{X}$ & $16.68 \pm 6.16$ & $22.68 \pm 7.87$ \\
\hline p-value & 0.333 & 0.333 \\
\hline \multicolumn{3}{|l|}{ Poor cooperation } \\
\hline $\mathrm{O}$ & $19.68 \pm 5.90$ & $26.08 \pm 8.48$ \\
\hline $\mathrm{X}$ & $15.27 \pm 5.34$ & $24.87 \pm 12.73$ \\
\hline p-value & 0.413 & 0.730 \\
\hline
\end{tabular}

Values are presented as mean \pm standard deviation.

NIHSS, National Institutes of Health Stroke Scale; NA, not applicable; OPA, oropharyngeal airway; MMSE, MiniMental State Examination; DM, diabetes mellitus; O, presence; $\mathrm{X}$, absence.
Table 4. $p$-values for SAGA-8 scores on the 3rd and 10th day, and the development of complications

\begin{tabular}{lcc}
\hline & Value & p-value \\
\hline SAGA-8 (on the 3rd day) & & \\
With OPA & $24.00 \pm 3.464$ & \\
\hline Without OPA & $20.80 \pm 2.950$ & 0.201 \\
SAGA-8 (on the 10th day) & & \\
With OPA & $25.50 \pm 2.385$ & \\
Without OPA & $21.40 \pm 3.130$ & 0.066 \\
\hline $\begin{array}{l}\text { Abnormal chest radiograph } \\
\text { Complications of tube feeding }\end{array}$ & 0.066 \\
\hline
\end{tabular}

Values are presented as mean \pm standard deviation.

SAGA-8, Satisfaction Questionnaire with Gastrostomy Feeding; OPA, oropharyngeal airway.

\section{Complications during IOET insertion}

Torsion during IOET insertion or incorrect insertion via the OPA during the VFSS occurred in 4 out of the 9 patients, whereas the tubes were inserted uneventfully in the remaining 5 cases.

Effects of OPA use on patient and caregiver satisfaction and associations with the medical history

To assess satisfaction with tube insertion via the OPA among the patients and caregivers, SAGA- 8 was administered 3 days after the initiation of IOET feeding, on the day oral feeding was initiated, on the day IOET feeding was stopped, or on the day of discharge. The use of the OPA did not result in significant differences in the SAGA8 scores obtained 3 days after the initiation of IOET feeding $(p=0.201)$. Similarly, there were no significant differences in the SAGA-8 scores obtained on the 10th day according to the OPA use ( $\mathrm{p}=0.066)$, although the patients/caregivers tended to be more satisfied with the procedure. Furthermore, no significant correlation was observed between insertion via the OPA and a history of other diseases, aspiration pneumonia on chest radiography, and diarrhea $(\mathrm{p}=0.054)$ (Table 4).

\section{DISCUSSION}

\section{Clinical effectiveness}

In the present study, we investigated the effects of OPA use on IOET insertion and assessed its application in dysphagic patients. In cases where changes in the scores for the functional swallowing scales were observed with OPA 
use, the time of passage from the mouth to the esophageal sphincter was reduced. Moreover, although no significant difference in caregiver and patient satisfaction was observed between the OPA and non-OPA groups 3 days after the start of IOET feeding as well as at later time points, the satisfaction tended to be higher in the OPA group on a longer follow-up. Based on these results, we believe that a decrease in the time of retention in the oral cavity likely contributes to higher satisfaction after OPA use. Statistical results showed a p-value $>0.05$; but to derive a broad interpretation in actual clinical practice, attention should be given to the measurement values with significance at the border range. One advantage of IOET feeding is the short feeding time. The time required for insertion of the IOET catheter was lower in the OPA group than in the non-OPA group, which suggests that the OPA could be useful for IOET feeding. It is possible that the reduced tube insertion time reflects the role of the OPA as a tongue depressor, and its role in reducing the posterior bolus transit time and inducing an early swallow reflex. The OPA also reduces resistance, which simplifies insertion of the IOET deep into the anterior pharynx while pushing the tongue to one side, thus alleviating delivery tube obstruction [15]. When the IOET reaches a certain insertion depth, the most commonly encountered problem is the loss of maneuverability of its advancing tip. This problem can be circumvented by forward displacement of the larynx. In particular, in patients with reduced consciousness, such as those in the present study, stylets have been used to reduce the passage time during IOET insertion [16]. Kisa et al. [16] developed a special catheter to facilitate the easy passage of the narrow rubber feeding catheter through the pharynx without any bending in the oropharynx. However, this stylet needs to be introduced within the IOET to increase its stiffness. Patients can ingest the OPA tube on their own to facilitate tube insertion (by swallowing it prior to insertion), without any help from caregivers or guardians. Moreover, by using the OPA, patients who have difficulty controlling their tongue can feed themselves through the IOET. The incidence of disuse syndrome is reduced in cases where the oral cavity, pharynx, and soft palate are directly stimulated by the catheter tip. In the present study, the use of the OPA along with the IOET tip could stimulate the oral soft tissues, promoting tactile facilitation [17].

In some cases, patients refused to undergo IOET inser- tion with the OPA or discontinued IOET feeding because the method was difficult to use or uncomfortable and unfamiliar. A previous study also reported that the IOET made the patients and caregivers feel uncomfortable and affected the patient's quality of life [15]. These perceived disadvantages of the OPA or IOET are more likely to be related to inadequate education on tube insertion rather than clinical side effects [6]. For instance, although in a previous study, 4 patients underwent repeated training on IOET insertion for 5 days and 1 of these patients actually inserted the IOET during hospitalization, nasogastric tube feeding had to be eventually initiated at the time of discharge owing to the inability to properly use the IOET [18]. Strong preference for the Levin tube feeding method may be the reason why many patients refused the OPA, as they had become accustomed to that method [15]. Similarly, in the present study, satisfaction with IOET feeding did not significantly differ depending on whether the OPA was used. However, given the observed tendency for higher satisfaction at a later time point in the study, a comparison of 3-day SAGA-8 scores and 10-day discharge SAGA-8 scores could reveal a more substantial difference between the OPA and non-OPA groups. Based on the current findings, we aim to develop a customized threedimensional guide canal and oral gavage to facilitate easy IOET insertion in the future.

\section{Study limitations}

The most significant problem in this study seems to be the small sample size, which reduces its statistical power. Furthermore, accurately positioning the IOET is more important than quickly performing the IOET procedure. Although the primary end point was not IOET insertion in a short period but rather safe insertion, no reference about safety assurance could be cited because this is the first study on this topic. In addition, the present study would have demonstrated better results if the safety assurance of the OPA had been verified in the pilot study. However, it was difficult to verify the safety of the OPA. Thus, the OPA was used instead in a pilot test with a $5 \%$ significance level and $\mathbf{8 0 \%}$ statistical power in order to estimate the time required for IOET insertion. It was found that 31 patients were required for the present study. In terms of per-protocol analysis, the overall frame was valid for demonstrating this evidence. However, in this study, more number of subjects dropped out than that predict- 
ed initially, and the resulting small sample size made it difficult to draw conclusions about the superiority of the OPA in terms of safety. The results of this study suggest that IOET insertion may be performed via the OPA and they also suggest the possible importance of satisfaction of patients and caregivers with IOET feeding. Second, this was a single-center study. Third, it was necessary to design the study so as to reduce the dropout rate by performing bedside screening tests to determine whether oral feeding was possible before conducting VFSS tests $[18,19]$. Altered vocal production after swallowing has been considered as a leading indicator of inefficient oral feeding, as stasis of food in the laryngeal-pharyngeal cavity during swallowing is commonly observed in patients with dysphagia [18]. In the oral feeding trial during the VFSS, most patients were considered to be capable of oral feeding, indicating that there was no need for IOET feeding. Hence, these patients were excluded, resulting in a small number of enrolled individuals. More patients should be included in future analysis. Fourth, although sufficient education on IOET insertion was provided to patients and caregivers, there is a possibility of selection bias. This may be due to the high dropout rate in the cases where SAGA-8 scores were recorded, given that tube feeding was stopped within 3 days after its initiation in many patients. The high dropout rate during the course of the study may be related to the unfamiliarity of the patients, caregivers, and doctors in other departments with the IOET method. Hence, it is essential to provide sufficient information on IOET insertion and management to eligible patients. Moreover, thorough management of patients and caregivers is required for long-term and safe application. None of the studies have assessed the reliability and validity of SAGA-8 scores. In addition, as the IOET can cause gastrointestinal problems similar to those caused by other enteral nutrition methods, it is essential to monitor gastrointestinal problems such as diarrhea, nausea, vomiting, and abdominal distension, and when necessary, it is essential to adjust the injection rate and utilize diluted supplements. Finally, the OPA size and location were not standardized, possibly leading to bias. A future randomized study on the OPA size with a larger number of patients may produce a more accurate result regarding the effects on swallowing and would help to prevent tube torsion. However, the benefits could exceed the potential hazards of inserting an IOET in patients with decreased alertness and cognitive impairment, such as the participants of this study. In other words, the OPA facilitates quick IOET insertion. In a broader sense, it is associated with a significantly higher SAGA-8 score in the long term. These findings can provide an important basis for a randomized controlled study in the future and can suggest possibilities for feeding methods other than percutaneous endoscopic gastrostomy (PEG) and Levin tube feeding in patients with impaired consciousness.

In conclusion, the use of the OPA for IOET insertion is feasible and it reduces IOET insertion time. Satisfaction of patients and caregivers with IOET feeding is an important factor.

\section{CONFLICT OF INTEREST}

No potential conflict of interest relevant to this article was reported.

\section{ACKNOWLEDGMENTS}

The authors would like to thank Dr. Dae Kwon Park and Dr. Woo Ram Koo for their assistance with VFSS.

\section{REFERENCES}

1. Smithard DG, O'Neill PA, Parks C, Morris J. Complications and outcome after acute stroke. Does dysphagia matter? Stroke 1996;27:1200-4.

2. Campbell-Taylor I, Nadon GW, Sclater AL, Fisher R, Harris-Kwan J, Rosen I. Oro-esophageal tube feeding: an alternative to nasogastric or gastrostomy tubes. Dysphagia 1988;2:220-1.

3. Gomes GF, Pisani JC, Macedo ED, Campos AC. The nasogastric feeding tube as a risk factor for aspiration and aspiration pneumonia. Curr Opin Clin Nutr Metab Care 2003;6:327-33.

4. Kim Y. Nutritional considerations in tube feeding patients. J Korean Dysphagia Soc 2011;1:85-9.

5. Han TR, Paik NJ, Park JW. The follow-up of OroEsophageal (OE) tube feeding. J Korean Acad Rehabil Med 2001;25:58-61.

6. Nakajima M, Kimura K, Inatomi Y, Terasaki Y, Nagano $\mathrm{K}$, Yonehara $\mathrm{T}$, et al. Intermittent oro-esophageal tube feeding in acute stroke patients: a pilot study. Acta Neurol Scand 2006;113:36-9. 
7. Han TR, Paik NJ, Park JW, Kwon BS. The prediction of persistent dysphagia beyond six months after stroke. Dysphagia 2008;23:59-64.

8. Davies JA, Maguire AM. Variability in Guedel airway design. Anaesthesia 2006;61:297-8.

9. Logemann JA, Gensler G, Robbins J, Lindblad AS, Brandt D, Hind JA, et al. A randomized study of three interventions for aspiration of thin liquids in patients with dementia or Parkinson's disease. J Speech Lang Hear Res 2008;51:173-83.

10. Kuhlemeier KV, Yates P, Palmer JB. Intra- and interrater variation in the evaluation of videofluorographic swallowing studies. Dysphagia 1998;13:142-7.

11. Kang Y, Na DL, Hahn S. A validity study on the Korean Mini-Mental State Examination (K-MMSE) in dementia patients. J Korean Neurol Assoc 1997;15:300-8.

12. Logemann JA. Manual for the videofluorographic study of swallowing. 2nd ed. Austin: Pro-Ed; 1993.

13. Martinez-Costa C, Calderon C, Pedron-Giner C, Borraz S, Gomez-Lopez L. Psychometric properties of the structured Satisfaction Questionnaire with Gastrostomy Feeding (SAGA-8) for caregivers of children with gastrostomy tube nutritional support. J Hum Nutr Diet 2013;26:191-7.

14. Best C. Caring for the patient with a nasogastric tube. Nurs Stand 2005;20:59-65.

15. Ozer S, Benumof JL. Oro- and nasogastric tube passage in intubated patients: fiberoptic description of where they go at the laryngeal level and how to make them enter the esophagus. Anesthesiology 1999;91: 137-43.

16. Kisa T, Igo M, Inagawa T, Fukada M, Saito J, Setoyama M. Intermittent oral catheterization (IOC) for dysphagic stroke patients. Jpn J Rehabil Med 1997;34:113-20.

17. Logemann JA. Evaluation and treatment of swallowing disorders. 2nd ed. Austin: Pro-Ed; 1998.

18. Song YJ, Park EJ, Yoon IJ, Park SJ. Tube feeding of dysphagia: the effect of oroesophageal tube feeding. J Korean Soc Occup Ther 2009;18:31-41.

19. Santos KW, Scheeren B, Maciel AC, Cassol M. Vocal variability post swallowing in individuals with and without oropharyngeal dysphagia. Int Arch Otorhinolaryngol 2015;19:61-6. 
Appendix 1. Satisfaction Questionnaire with Gastrostomy Feeding (SAGA-8)

\section{Satisfaction Questionnaire with Gastrostomy Feeding 8 (SAGA-8)}

\begin{tabular}{|l|}
\hline Question (Q) \\
\hline \\
\hline Q1 : How do you rate your satisfaction with GT feeding? \\
\hline Q2: How do you evaluate GT management? \\
\hline Q3 : How do you evaluate the support offered by our center? \\
\hline Q4: How do you perceive your child's change in nutritional status? \\
\hline Q5: How do you rate the change in your child and your family's overall situation? \\
\hline Q6: Has the time required for feeding decreased? \\
\hline Q7: Has the number of respiratory infections decreased? \\
\hline Q8: Would you accept earlier GT placement with your current knowledge of the benefits of the procedure? \\
\hline
\end{tabular}

Q1 : Score range from 1 (totally unsatisfied) to 5 (very satisfied).

Q2 : Score range from 1 (very difficult) to 5 (very easy).

Q3 : Score range from 1 (totally insufficient) to 5 (very satisfactory).

Q4 : Score range from 1 (deteriorated) to 5 (significantly improved).

Q5 : Score range from 1 (deteriorated) to 5 (significantly improved).

Q6, 7,8: Score range from 1 (no) to 2 (yes).

GT : gastronomy tube. 
Appendix 2. Intermittent oroesophageal tube (IOET) feeding

\section{Intermittent oroesophageal tube (IOET) feeding}

Preparation materials: oropharyngeal airway (OPA, \#3, \#4, \#5), Nelaton rubber catheter (12, 14, $16 \mathrm{Fr})$, and 30-mL syringe

1. Dip one tip of the Nelaton rubber catheter into water in order to achieve smooth insertion.

2. After testing the gag reflex and comparing the two sides, push the OPA onto the base of the tongue on the side with a weaker reflex.

3. Remove the OPA immediately if cough, severe gag reflex, or dyspnea is present.

4. Insert the Nelaton rubber catheter slowly into the OPA orifice.

5. After inserting the Nelaton rubber catheter, check whether the catheter has folded within the mouth or whether it has proceeded into the airway by using fluoroscopy.

6. Remove the OPA immediately if cough, severe gag reflex, or dyspnea is present.

7. If the tube is inserted safely, mix $140 \mathrm{~g} / \mathrm{mL}$ of liquid barium sulfate with $300 \mathrm{~mL}$ of normal saline, dilute this solution to $40 \% / \mathrm{v}$, transfer it into a $30-\mathrm{mL}$ syringe, and inject $10 \mathrm{~mL}$. Thereafter, check the reflux.

Size of the intermittent oroesophageal tube catheters

\begin{tabular}{cccc}
\hline & $12-\mathrm{Fr}$ & $14-\mathrm{Fr}$ & $16-\mathrm{Fr}$ \\
\hline Diameter & $4 \mathrm{~mm}$ & $4.6 \mathrm{~mm}$ & $5.3 \mathrm{~mm}$ \\
\hline
\end{tabular}

Total length, $400 \mathrm{~mm}$. Manufactured by Nantong Angel Medical Instruments. Co., Ltd (China).

Size of the oropharyngeal airway used for intermittent oroesophageal tube feeding

\begin{tabular}{lrrr}
\hline & $\# 3$ & $\# 4$ & $\# 5$ \\
\hline Total length & $930 \mathrm{~mm}$ & $1,000 \mathrm{~mm}$ & $1,150 \mathrm{~mm}$ \\
Outer diameter of the airway & $360 \mathrm{~mm}$ & $400 \mathrm{~mm}$ & $400 \mathrm{~mm}$ \\
Inner diameter of the airway & $300 \mathrm{~mm}$ & $300 \mathrm{~mm}$ & $300 \mathrm{~mm}$ \\
Outer diameter of the small opening & $180 \mathrm{~mm}$ & $180 \mathrm{~mm}$ & $180 \mathrm{~mm}$ \\
Inner diameter of the small opening & $7 \mathrm{~mm}$ & $7 \mathrm{~mm}$ & $7 \mathrm{~mm}$ \\
\hline
\end{tabular}

The internal diameter is approximately $50 \mathrm{~mm}$, and the general angle is 105 degrees. The OPA was manufactured by Solco Biomedical Co. (Pyungtaek, Korea). 
Appendix 3. Instructions on feeding using the intermittent oroesophageal tube (IOET)

\section{Instructions on feeding using the intermittent oroesophageal tube (IOET)}

1. Once the patient/caregiver is educated about the safe use of the IOET, feeding can be initiated.

2. During feeding, fix the tip of the IOET to the left side after the insertion of a ball, and adjust the flow of the food to $20 \mathrm{~mL} / \mathrm{min}$. For intake of water or medicine, connect a $50-\mathrm{mL}$ syringe without a piston to the IOET, transfer the contents into the syringe, and lift it above the oral cavity.

3. After feeding is completed, wait for the leftovers in the IOET to flow into the esophagus, and then remove the IOET.

4. Wash the used IOET under running water and disinfect the tube in diluted vinegar $(50 \mathrm{~mL}$ of vinegar in $200 \mathrm{~mL}$ of water). Discard the tube after using it for 2 days. 